\title{
Jamming and unjamming of concentrated colloidal dispersions in channel flows $\dagger$
}

\author{
Andrew I. Campbellt* and Mark D. Haw \\ Received 15th March 2010, Accepted 29th June 2010 \\ DOI: 10.1039/c0sm00110d
}

\begin{abstract}
We investigated the pressure driven flow of concentrated colloidal dispersions in a converging channel geometry. Optical microscopy and image analysis were used to track tracer particles mixed into dispersions of sterically stabilized poly(methyl methacrylate) (PMMA) spheres. The dispersions were drawn into a round $0.5 \mathrm{~mm}$ capillary at one of two pump speeds (三 applied pressure): $v_{1}=0.245 \mathrm{ml}$ $\min ^{-1}$ and $v_{2}=0.612 \mathrm{ml} \mathrm{min}^{-1}$. We observed that the dispersions at particle volume fractions $\phi \leqslant 0.50$ followed Hagen-Poiseuille flow for a simple fluid; i.e. the mean flow rate $\langle V\rangle$ is approximately proportional to pressure drop (pump speed) and inversely proportional to viscosity $\eta$. Above this concentration $(\phi \geqslant 0.505)$, the dispersions exhibit granular-like jamming behaviour with $\langle V\rangle$ becoming independent of the pressure drop. However, at the highest applied pressure $\left(v_{2}\right)$, the dispersions are able to unjam and switch from granular-like behaviour back to a simple hard-sphere liquid like system, due to the formation of rotating vortices in the spatial flow pattern. This mechanism is consistent with computer simulations of granular systems and supports for example proposed explanations of anomalously low friction in earthquake faults. We also link this behaviour with the concept of fragile matter (M. E. Cates, J. P. Wittmer, J. P. Bouchaud and P. Claudin, Phys. Rev. Lett., 1998, 81, 1841-1844).
\end{abstract}

\section{Introduction}

Understanding and controlling the flow and deformation of particle suspensions through confined geometries is of significant technological importance. ${ }^{2-4}$ Soft matter materials consisting of colloidal particles or droplets suspended in a liquid medium are frequently encountered in industrial products and applications. e.g. foods, paints, building materials, pharmaceuticals. Processing of such concentrated colloidal dispersions often involves driving under pressure through complex geometries, namely convergent and divergent pipe sections, generating extensional components of strain. ${ }^{5}$ Under suitably low shear rates concentrated dispersions exhibit a constant viscosity; i.e. they behave as a Newtonian fluid. ${ }^{6}$ However, under higher stress or shear rate they can also exhibit shear thickening 7 i.e. increase in viscosity with stress. Extreme or 'discontinuous' shear thickening has been reported where apparent viscosity increases very suddenly, accompanied by subsequent large fluctuations in viscosity and stress. $^{7,8}$ Such extreme thickening has been linked with flowinduced jamming. ${ }^{1}$ Jamming can be defined as the transformation of a liquid system to a solid by an applied stress. Whilst there have been some studies of concentrated colloidal systems under Poiseuille flow, ${ }^{5}$ the conditions under which jamming occurs are still not properly understood.

In this paper we demonstrate experimentally a novel behaviour of concentrated jamming suspensions, i.e. a 'self-lubrication'

Chemical and Process Engineering, The University of Strathclyde, James Weir Bld., 75 Montrose Street, Glasgow, G1 1XJ, United Kingdom. E-mail: andrew.campbell.103@strath.ac.uk

$\dagger$ Electronic Supplementary Information (ESI) available: Two video files are available for download showing the vortices at $\phi=0.505$ (movie_1) and $\phi=0.52$ (movie_2) at pump speed $v_{2}$. See DOI: $10.1039 / \mathrm{c} 0 \mathrm{sm} 00110 \mathrm{~d} /$ \$ Current address: NCPO, The University of Strathclyde, Glasgow, U.K effect that enables a jamming suspension to revert to an unjammed simple fluid flow behaviour. Associated with this is the creation of rotating vortex-like flow patterns, consistent with effects seen in computer simulations of granular systems but not to our knowledge observed before in experiment. ${ }^{9}$

\section{Experimental}

We study the pressure-driven flow of a concentrated model suspension, consisting of a reservoir of poly(methyl methacrylate) (PMMA) spheres sterically-stabilized by chemically grafted poly(12-hydroxystearic acid), dispersed in a refractive index matched mixture $(\mathrm{n}=1.51)$ of decalin and tetralin solvents. We determined the radius $(a)$ of the spheres to be $670 \mathrm{~nm}$ with a polydispersity $(\sigma)$ of $6.0 \%$ by static light scattering. We characterize the reservoir sphere concentration using the volume fraction parameter $\phi$, where $0.46 \leqslant \phi \leqslant 0.52$. The volume fraction is calculated from the dry weight of the dispersions using the density of PMMA $\left(\rho=1.188 \mathrm{~g} \mathrm{~cm}^{-3}\right)$ and the solvent $\left(\rho_{\mathrm{s}}=\right.$ $0.9375 \mathrm{~g} \mathrm{~cm}^{-3}$ ). Sterically stabilized PMMA particles suspended in simple hydrocarbon solvents, have received extensive study by the scientific community over the last 30 years. This is primarily because of the excellent approximation the system makes to the hard sphere model often used by theoreticians and simulationists. The phase behaviour ${ }^{10}$ and rheology of PMMA dispersions are therefore, well known. Where the particles are reasonably monodisperse $(\sigma \leqslant 8 \%)$ and the sample is left to stand, crystallization will occur at particle concentrations above $\phi=0.494$. Above $\phi \sim 0.58$ the system crosses in to a glass phase, where diffusion is suppressed and the dispersion remains amorphous because there is no longer sufficient free space for the particles to reorganize. Under low shear the viscosity of PMMA dispersions has been shown to rise rapidly as $\phi$ nears $0.5 .^{11}$ 
The sphere reservoir was contained in a glass cuvette measuring $2 \mathrm{~mm} \times 10 \mathrm{~mm} \times 40 \mathrm{~mm}$, mounted on an optical microscope stage so that the dispersion was observed along the 2 mm deep path, using a $\times 4$ (N.A. $=0.10$ ) objective. We inserted one end of a $5 \mathrm{~cm}$ length of a cylindrical $0.5 \mathrm{~mm}$ bore glass capillary into the reservoir, positioned so that it was approximately $0.5 \mathrm{~mm}$ from the top and bottom walls of the cuvette and about $4.5 \mathrm{~mm}$ from the side walls. The capillary was attached to a $2 \mathrm{ml}$ glass syringe via a $10 \mathrm{~cm}$ length of $1.0 \mathrm{~mm}$ bore PTFE tubing. Fig. 1 illustrates our experimental setup. A DC motor was used to draw the syringe plunger out reducing the air pressure in the syringe barrel. This effectively applies a pressure drop across the dispersion, between atmospheric pressure above the sample and reduced pressure in the barrel. The effective pressure drop applied was controlled by changing the pump motor speed. Results presented here focus on two pressure drops, with corresponding constant pump speeds $v_{1}=0.245 \mathrm{ml} \mathrm{min}^{-1}$ and $v_{2}=$ $0.612 \mathrm{ml} \mathrm{min}^{-1}$. These parameters correspond to average flow velocities as given in Tables 1 and 2; of course there is significant flow speed variation across the flow geometry and over time (see below) hence we differentiate experiments based on these pump speeds rather than actual particle flow rates.

An important question is how typical flow speeds compare to particle transport by diffusion. With the solvent viscosity $(\eta=$ $3.0 \mathrm{mPa} \mathrm{s})$, temperature $(T=293 \mathrm{~K})$ and particle size $a=670 \mathrm{~nm}$ relevant to these experiments, and using the Stokes Einstein relation for free particle diffusivity, a Brownian time for free diffusion of a particle by a distance equal to its radius is estimated at $\tau_{0} \sim 4 \mathrm{~s}$. However, in concentrated suspensions diffusion rates are greatly reduced:12,11 assuming a factor of $\sim 50$ slowdown, we have a Brownian time $\tau_{c} \sim 200 \mathrm{~s}$. With particle flow speeds on the order of $\sim 100 \mu \mathrm{m} \mathrm{s}^{-1}$ this means flow transport dominates diffusion. Alternatively, with typical local shear rates $\gamma \sim 0.1$ as measured from particle tracking results (see below), we can define a Peclet number (the ratio of shear to diffusive transport) $P e=\gamma \tau_{c} \sim 20$, i.e. shear typically dominates diffusion. It is important to remember, however, that flow rates and
Table 1 The average flow rate $\langle V\rangle$ for samples drawn in to the capillary using a syringe pump speed of $v_{1}=0.245 \mathrm{ml} \mathrm{min}^{-1}$. Average flow rates are calculated in three ways for comparison: over the full image area (col. 2), within the rectangular entrance zone indicated on the vector plots (col. 3), and over the area excluding the entrance zone ('anti-zone', col. 4)

\begin{tabular}{llll}
\hline$\phi$ & $\begin{array}{l}\langle V\rangle \\
\left(\mu \mathrm{m} \mathrm{s}^{-1}\right)\end{array}$ & $\begin{array}{l}\text { Entrance zone }\langle V\rangle \\
\left(\mu \mathrm{m} \mathrm{s}^{-1}\right)\end{array}$ & $\begin{array}{l}\text { Anti-Zone }\langle V\rangle \\
\left(\mu \mathrm{m} \mathrm{s}^{-1}\right)\end{array}$ \\
\hline 0.46 & $128 \pm 39$ & $214 \pm 65$ & $106 \pm 35$ \\
0.47 & $134 \pm 42$ & $222 \pm 51$ & $115 \pm 44$ \\
0.50 & $93 \pm 26$ & $169 \pm 46$ & $70 \pm 21$ \\
0.505 & $64 \pm 10$ & $115 \pm 21$ & $50 \pm 08$ \\
0.51 & $42 \pm 09$ & $85 \pm 22$ & $32 \pm 06$ \\
0.52 & $41 \pm 16$ & $78 \pm 40$ & $30 \pm 10$ \\
\hline
\end{tabular}

Table 2 The mean flow rate $\langle V\rangle$ for samples drawn in to the capillary using a syringe pump speed of $v_{2}=0.612 \mathrm{ml} \mathrm{min}^{-1}$. Samples which exhibited vortices are indicated $\left(^{*}\right)$ and $\langle V\rangle$ prior to the emergence of the vortices are given in brackets

\begin{tabular}{|c|c|c|c|}
\hline$\phi$ & $\begin{array}{l}\langle V\rangle \\
\left(\mu \mathrm{m} \mathrm{s}^{-1}\right)\end{array}$ & $\begin{array}{l}\text { Entrance zone }\langle V\rangle \\
\left(\mu \mathrm{m} \mathrm{s}^{-1}\right)\end{array}$ & $\begin{array}{l}\text { Anti-Zone }\langle V\rangle \\
\left(\mu \mathrm{m} \mathrm{s}^{-1}\right)\end{array}$ \\
\hline 0.46 & $243 \pm 36$ & $334 \pm 66$ & $222 \pm 44$ \\
\hline 0.47 & $350 \pm 49$ & $396 \pm 67$ & $342 \pm 50$ \\
\hline 0.50 & $201 \pm 30$ & $300 \pm 53$ & $180 \pm 35$ \\
\hline${ }^{*} 0.505$ & $104 \pm 25(106)$ & $241 \pm 39(198)$ & $71 \pm 23(82)$ \\
\hline${ }^{*} 0.51$ & $138 \pm 22(74)$ & $220 \pm 32(120)$ & $127 \pm 24(57)$ \\
\hline${ }^{*} 0.52$ & $124 \pm 33(31)$ & $275 \pm 62(77)$ & $90 \pm 30(21)$ \\
\hline
\end{tabular}

shear rates vary across the geometry and over time, and particularly where the flow is jammed, short-distance diffusion may still play an important role, for example in breaking up particle force chains.

During an experiment the pump was switched on and a sequence of time-stamped microscope images was recorded at a rate of $\sim 11 \mathrm{~Hz}$. Shown in Fig. 1 is a typical microscope image, with the expected flow pattern for a simple Newtonian liquid indicated. Image analysis algorithms were used to track tracer

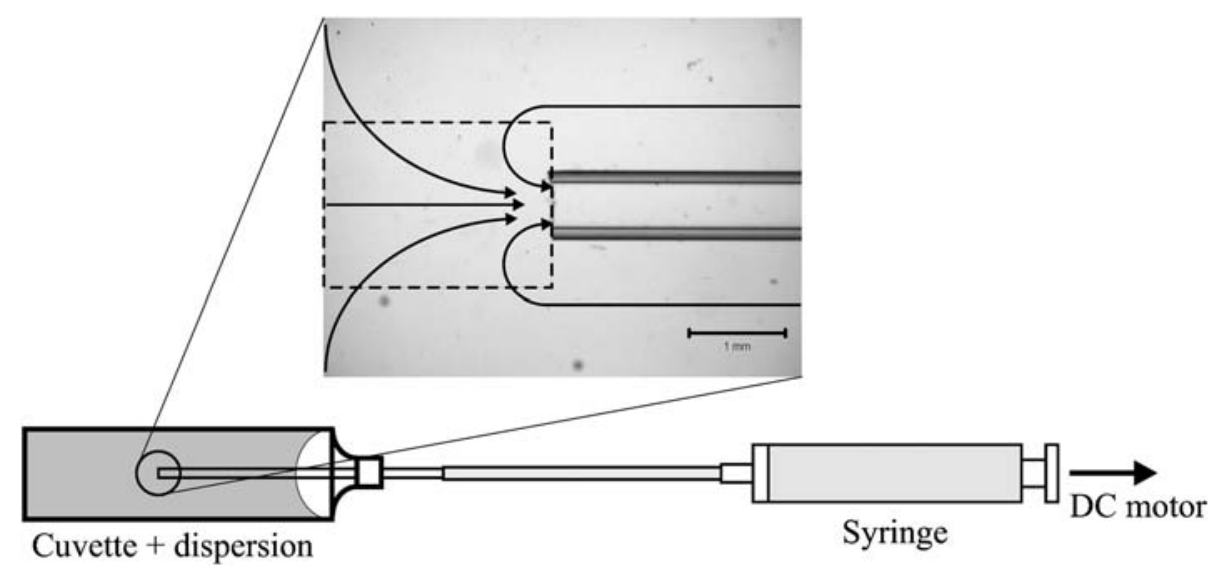

Fig. 1 Our experimental setup consisted of a glass cuvette filled with an index matched PMMA particle dispersion and a glass capillary attached to a syringe using PTFE tubing. A DC motor drew the syringe plunger out, applying a pressure drop across the dispersion drawing it into the capillary. The magnified view is a typical recorded image of a capillary immersed in a dispersion of PMMA particles $(\phi=0.46)$. The dashed-line rectangle defines an entrance zone, immediately in front of the capillary: we report average speeds across the whole imaged geometry as well as averages calculated within only this zone and excluding this zone (see Tables). The arrows indicate the expected flow pattern for a Newtonian liquid, flowing into the capillary from all directions (including above and below the image plane). 
particles between images ${ }^{13}$ and measure flow velocities. Tracer particles are either melamine spheres or simply particles of dust, at low concentration (tracers relative to PMMA particles $<0.1 \%$ by number) so they do not affect the flow, easily observed in transmission microscopy due to the close refractive index match of the PMMA particles. The tracer particles appeared as dark grey blobs on a light grey background and were followed by computing the centre of mass coordinates of the blobs ${ }^{14}$ after an initial thresholding step. We estimate the accuracy of tracking by measuring fluctuation in velocities measured by the tracking algorithm for a non-flowing system, ie where the particles should be subject only to diffusion. From this we obtain a position accuracy in a given frame of $\sim 2 \mu \mathrm{m}$. Fig. 1 also defines an entrance zone immediately in front of the capillary opening: average velocities (see below) are calculated both across the whole geometry and within this entrance zone. (Error bars in average velocities reported in the Tables are derived from variation across the population of measured tracer particles.)

\section{Results and discussion}

\subsection{Observations at $v_{1}=0.245 \mathrm{ml} \mathrm{m^{-1 }}$}

First we discuss our qualitative observations of the dispersions drawn into the capillary using the pump speed $v_{1}$; i.e. at the lowest applied pressure. The dispersions followed the spatial flow directions indicated in Fig. 1, with the rate at which the dispersion is drawn into the capillary decreasing as $\phi$ is increased. Previously, we have made qualitative observations of erratic flow and transient jamming in similar experiments of concentrated suspensions of PMMA spheres being drawn into a $1 \mathrm{~mm}$ capillary. ${ }^{5}$ Here, we begin to observe 'occasional' jamming at $\phi=0.51$ : in a typical experiment, about $20 \mathrm{~s}$ after turning on the pump the flow of the dispersion almost stops; the dispersion immediately in front of the capillary continues to be drawn in at a very slow rate. The jammed state persists for about $15 \mathrm{~s}$ before flow suddenly resumes. No further jamming was observed at this sphere concentration for the remainder of the experiment ( $300 \mathrm{~s})$. However, by $\phi=0.52$ we observe repeated periods of flow followed by jammed states throughout the typical experiment.

To quantify this flow-jamming behaviour we first computed the mean flow rate $\langle V\rangle$ of the tracer particles as the suspensions are drawn into the capillary. In Table 1 we show $\langle V\rangle$ for dispersions being drawn in to the capillary at the pump speed $v_{1}$; the flow rate for tracer particles passing through the entrance zone indicated in Fig. 1, outside of the zone and over the full image frame are given. As $\phi$ increases $\langle V\rangle$ decreases, not surprising as it is well known that the viscosity of a dispersion of nearly hard spheres increases markedly with $\phi .^{12,11}$

A feature that was not evident in qualitative observations is the long period oscillation $(\sim 30 \mathrm{~s})$ of the flow rate which we begin to observe for $\phi \geqslant 0.505$. This behaviour is most clearly seen in the $\phi=0.505$ plot (see Fig. 2(a), solid line). These oscillations are consistent with previous observations such as density waves. ${ }^{5} \mathrm{~A}$ proposed explanation is that particle configurations temporarily approach jamming, i.e. transmit some fraction of applied pressure drop via force chains to the walls of the geometry, leading to a slowdown in $\langle V\rangle$; however the pressure gradient continues to drive solvent through the pores of the jam, dilating (decreasing

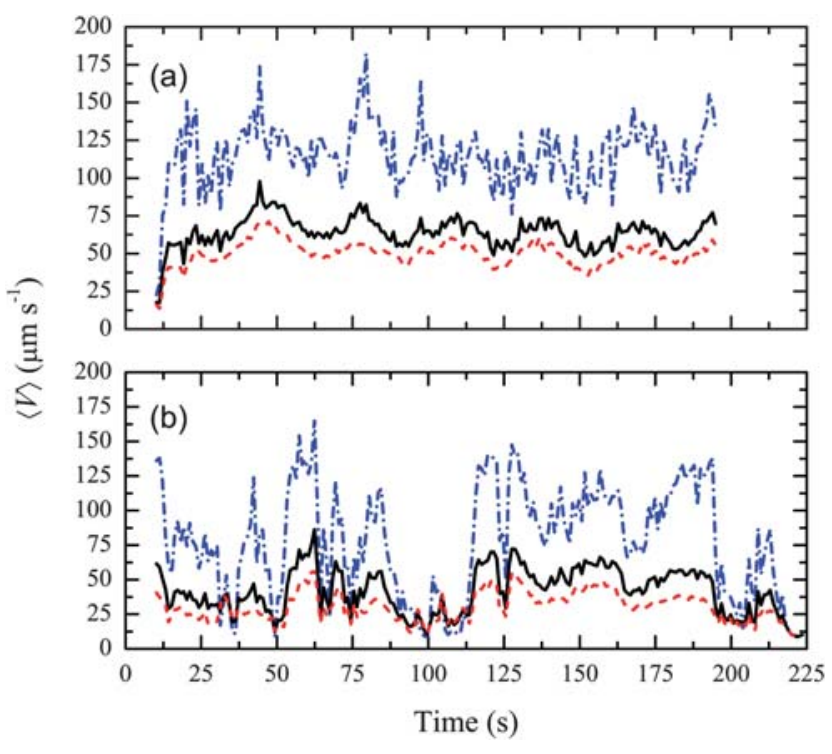

Fig. 2 Mean flow rates $\langle V\rangle$ for dispersions drawn into the capillary using

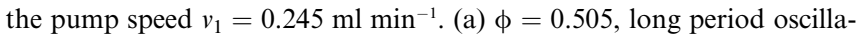
tions $(\sim 30 \mathrm{~s})$ are clearly visible in the flow rate. (b) $\phi=0.52$, the dispersion cycles through numerous flow-jam-flow states. Both plots: $(-)$ $\langle V\rangle$ over full image area, (-.-) $\langle V\rangle$ within the entrance zone, $(--)\langle V\rangle$ outside of the zone (anti-zone).

$\phi)$ downstream of the jam and enabling unjamming. A recent study by Isa et al. $^{15}$ of a similar system under pressure-driven flow but this time in a micro-channel also showed high frequency oscillations above a critical driving pressure, with a similar proposed explanation combining fluid permeation and jamming of the particles.

As $\phi$ increases the clear periodic oscillations transform into irregular and significantly long-lived jams followed by sudden unjamming (e.g. $\phi=0.52$, Fig. 2(b)). The mechanics of the hardsphere flow is thus highly sensitive to $\phi$, transforming from constant regular flow to oscillatory behaviour to erratic jamcollapse behaviour in a two percent range of volume fraction. Note that if fluid permeation is involved, i.e. differing flow rates of particle and fluid phase, then spatial and temporal local volume fraction variations are expected (dilation and compression); however, even by tracking every particle with confocal microscopy ${ }^{15}$ direct measures of local $\phi$ to the required accuracy are very challenging, since a precise measure of the size of every particle is required. On the other hand bulk changes in downstream $\phi$ due to dilation are easily measured. ${ }^{5}$

In Fig. 3(a) we plot the spatial map of velocity for the sample at $\phi=0.505$, at the lowest applied pressure (pump speed $v_{1}$ ). The velocity map is averaged over a period of $185 \mathrm{~s}$, with the magnitude of the vector arrows encoded in their colour (online). The velocity map follows that indicated in Fig. 1 for a Newtonian liquid. In fact, velocity maps for all $\phi$ at pump speed $v_{1}$ follow the same pattern. Only the magnitude of the vectors is seen to decrease. The flow-jam-flow behaviour observed at $\phi=0.52$ is not resolved in the velocity map. We conclude that at this applied pressure drop, despite generating temporal fluctuations in local pressure, jamming does not change the time-averaged spatial flow pattern. 

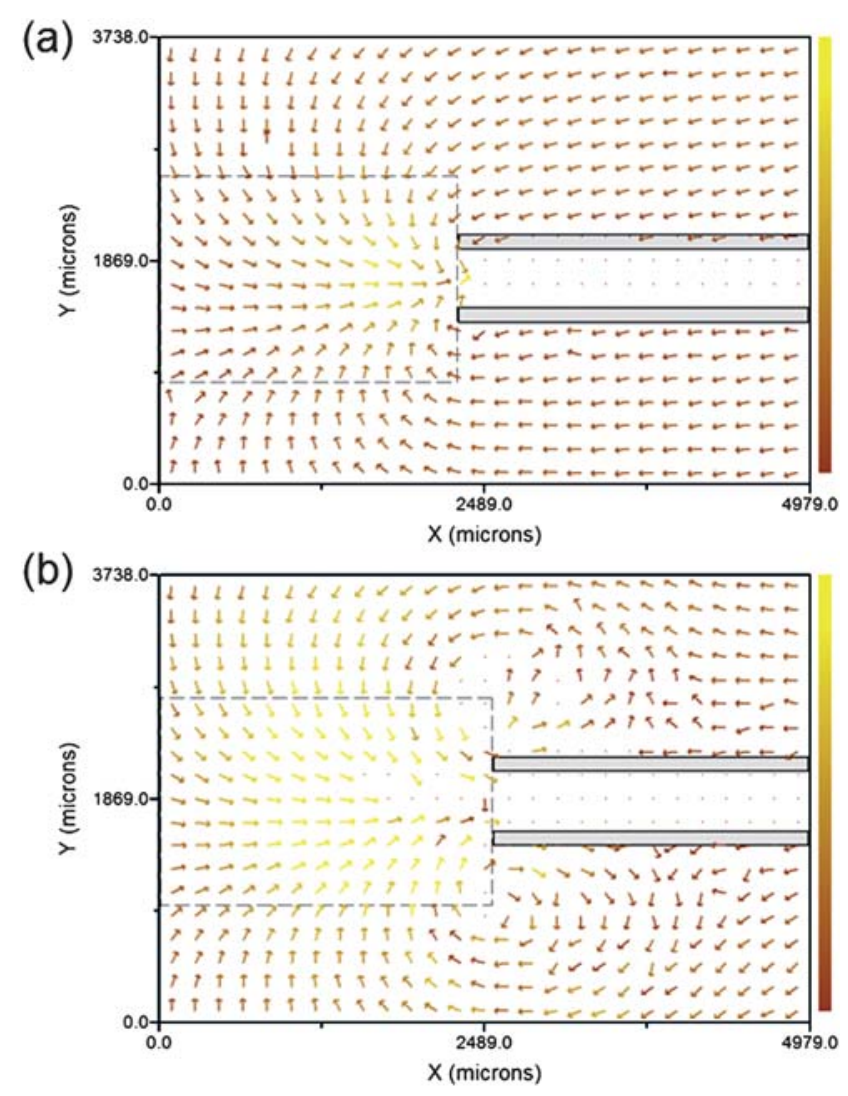

Fig. 3 (a) Spatial velocity map for the PMMA concentration $\phi=0.505$ and pump speed $v_{1}$. The dispersion follows the flow pattern expected of a Newtonian liquid. (b) The spatial velocity map for the PMMA concentration $\phi=0.505$ and pump speed $v_{2}$, illustrating the vortex flow pattern on both sides of the capillary entrance. The vortices formed about $20 \mathrm{~s}$ after switching on the pump. The colour bar (online) indicates a velocity range of $0 \mu \mathrm{m} \mathrm{s}^{-1}$ to $300 \mu \mathrm{m} \mathrm{s}^{-1}$.

\subsection{Observations at $v_{2}=0.612 \mathrm{ml} \mathrm{min}^{-1}$}

We now compare this flow behaviour with that at the higher applied pressure drop (pump speed $v_{2}$ ). In Table 2 the flow rates of the tracer particles being drawn into the capillary under the higher applied pressure are given.

We can divide the flow behaviour at $v_{2}$ into two distinct groups. The first group features the dispersions where $\phi \leqslant 0.50$. In this group we observed the same flow pattern as that seen at $v_{1}$; i.e. the dispersions followed the time-averaged flow pattern for a Newtonian liquid. Due to the higher applied pressure the dispersions were drawn into the capillary at a higher rate. A decrease in $\langle V\rangle$ is again seen with increasing $\phi$.

In the second group $(\phi \geqslant 0.505)$, however, we observed a significant change in the flow behaviour of the dispersions. At first the dispersions exhibited the same velocity field as for the samples $\phi \leqslant 0.50$. After this initial period the velocity field changed. Fig. 3(b) shows the velocity map for $\phi=0.505$ averaged over a period of $100 \mathrm{~s}$, starting from $20 \mathrm{~s}$ after the pump $\left(v_{2}\right)$ was switched on. Vortices (ESI $\dagger$ ) are evident on either side of the capillary opening resulting in the reversal of flow along the outside of the capillary, extending back about $1 \mathrm{~mm}$. Vortices appear for all $\phi \geqslant 0.505$, the distance that the vortices extend back along the capillary increasing with $\phi$. Once formed, the vortices are permanent. It is not clear what determines the timescale of the vortex formation, though this may depend on the rate of permeation of fluid through the initially jammed (pre-vortex formation) particle structure: in future work this will be tested by changing the fluid viscosity. At the top of the image the vortex rotates in an anticlockwise direction whilst at the bottom of the image the vortex rotates in a clockwise direction. Tracer particles trapped in the rotating mass may go through three or four revolutions before being drawn into the capillary, indicating that there is some exchange of PMMA particles in the rotating mass and the surrounding dispersion. The vortices appear to be spatially separate. However we are unable to reliably measure velocities out of the focal plane in this flow geometry. Therefore based on what measurements are possible, we do not rule out the possibility that there may be a single toroidal vortex encircling the capillary entrance out of the plane of the image.

Associated with the formation of the vortices the flow rate of the dispersion shows a significant increase. In Fig. 4(a) we plot $\langle V\rangle v$ s. time for the $\phi=0.52$ dispersion. An arrow indicates the moment at which the vortices begin to form, immediately after which the flow rate rises rapidly and approaches the value seen for $\phi=0.50$.

In Fig. 4(b) we plot $\langle V\rangle$ for the dispersions passing through the entrance zone against $\phi$, for the two applied pressure drops (pump speeds $v_{1}$ and $v_{2}$ ). For $\phi \leqslant 0.50\langle V\rangle$ changes across the range of $\phi$ by similar factors of $\sim 1.3$ at both $v_{1}$ and $v_{2}$. This is comparable to the change in viscosity $(\eta)$ expected for a hardsphere system across this $\phi$-range. Additionally, at fixed $\phi,\langle V\rangle_{v_{1}} I$ $\langle V\rangle_{v_{2}} \sim v_{1} / v_{2}$, i.e. overall flow speed is approximately proportional to pressure drop (三 pump speed). We conclude from this that the

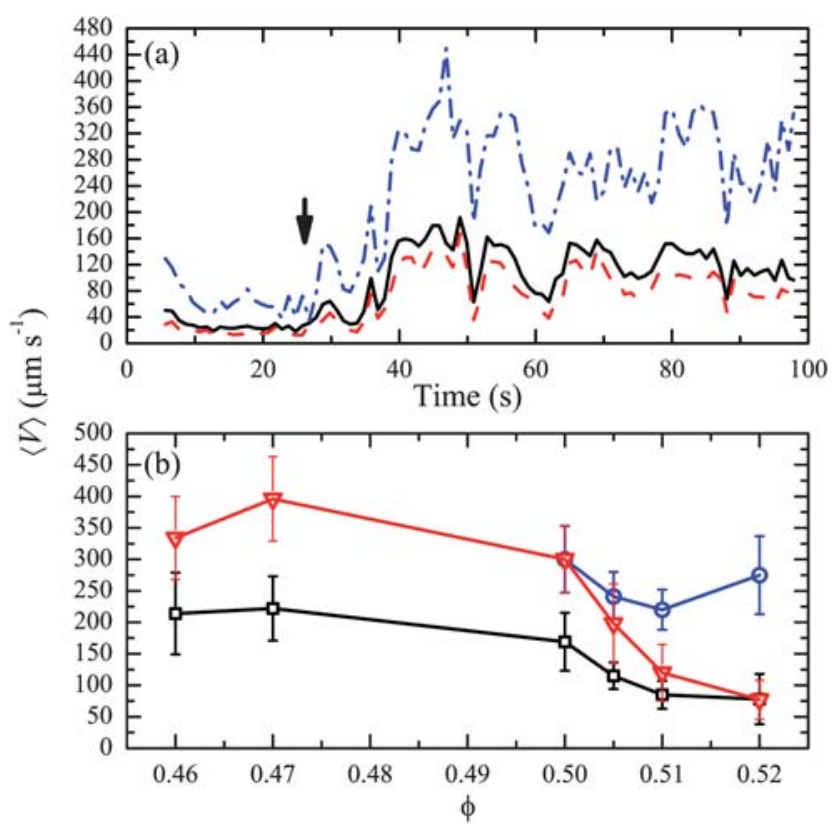

Fig. 4 (a) Mean flow rate $\langle V\rangle$ for the dispersion $\phi=0.52$ and a pump speed of $v_{2}=0.612 \mathrm{ml} \mathrm{min}^{-1}$. The arrow indicates the time when the vortices were first observed. See Fig. 2 for key. (b) Mean flow rates $\langle V\rangle$

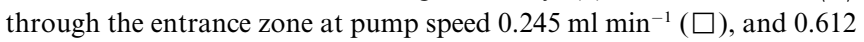

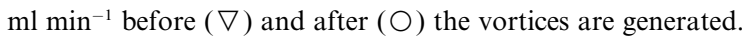


$\langle V\rangle$ data for $\phi \leqslant 0.50$ is approximately consistent with HagenPoiseuille flow of a simple fluid, namely $\langle V\rangle \propto$ pressure drop (三 pump speed) at fixed $\phi$ and $\langle V\rangle \propto \eta^{-1}$ at a fixed pressure drop. ${ }^{6,11}$ For $\phi \leqslant 0.50$ the suspensions effectively flow as a simple hardsphere fluid.

However, for $\phi \geqslant 0.505$, at pump speed $v_{1}$ and before the formation of vortices at pump speed $v_{2}$, the overall flow rate decreases more rapidly with $\phi$. By $\phi=0.52$ the flow rates for the two pump speeds (applied pressure drops) coincide; i.e. flow rates become independent of pressure drop. Hence at $\phi \geqslant 0.505$ flow behaviour is characteristic of granular systems, for example grains in a hopper or sand-timer, where the flow rate is independent of load pressure. Extra applied pressure, rather than increasing flow, is transferred to the walls of the system by force chains or blocking arches. ${ }^{2}$

The above granular-like behaviour applies before the formation of vortices in the spatial flow pattern. Surprisingly, the dispersions drawn into the capillary at the highest pump speed $v_{2}$ (三 higher applied pressure) appear to be able to switch from this granular-like flow back to mean flow rates that are indicative of simple hard-sphere fluids, through the formation of vortices. As shown in Fig. 4(b), after the vortices form $\langle V\rangle$ reverts to speeds approximately proportional to pressure drop (pump speed) once again, i.e. simple fluid flow behaviour. The vortices allow the jammed, granular-like fluid to revert to simple viscous fluid, i.e. the fluid 'self-lubricates' to escape jamming.

We qualitatively explain the vortex and self-lubrication effect as follows. In a jamming granular or colloid in a straight channel, force chains form that transmit upstream applied pressure to the channel walls, shielding downstream particles from pressure increase and leading to a flow speed independent of increases in pressure. However, in the geometry studied here the change in direction of flow downstream tends to rotate the force chain at the capillary outer wall. The effect of this rotation is to establish the vortex near the end of the capillary wall, preventing formation of stable force chains and transmission of pressure to the capillary wall. The flowing suspension therefore does not jam, passing the rotating vortex region with reduced shear and reverting from jammed granular-like to simple viscous fluid where flow rate is proportional to applied pressure drop.

The unjamming can also be interpreted as an example of socalled fragile matter behaviour. ${ }^{1}$ A fragile system is jammed in response to a force in a given direction but not jammed in other directions. Here, the directions of the jamming pressure and the centripetal force providing the turn in the flow differ, hence the system behaves as 'fragile' and is able to unjam.

Formation of rotating vortices in granular shear and resulting reduction in shear stress or friction has been proposed as an explanation of anomalously low observed or inferred friction in granular processes such as earthquake deformation. Such effects have been seen in computer simulations ${ }^{9}$ but not to our knowledge in experimental systems, granular or colloidal; nor have they been related to the fragile matter concept.

Some initial support for the idea that vortices result from the rotation of force chains comes from a consideration of timescales observed in the experiments. Based on typical flow rates/shear rates measured by particle tracking in the region just upstream of where vortices form, during the 'induction' period before we observe the vortex-like flow, we find that a force chain would rotate such as to give a strain $\gamma \sim 1$ (i.e. by $\sim 45^{\circ}$ ) in a time of the same order as the induction time $(\sim 10-20 \mathrm{~s})$ : in other words the delay before vortices form is the time taken for an initially formed jamming force chain to rotate due to the 'turn' in the flow. Once rotated the chain can no longer resist the overall pressure gradient, and instead establishes the vortex pattern. Once established, the rotating 'bearing' inhibits further formation of jamming chains upstream, and the transition to non-jamming flow is established. This explanation clearly requires further direct measurements to verify the details, which are the subject of current work.

Finally we offer a speculative comment on why vortices do not form at the lower pressure drop in these experiments, again based on a consideration of flow speeds and timescales. With slower flow at lower pressure drop, the time required for travel through the 'turning' region of the flow (rotation of a force chain) is longer: for example at $\phi=0.505$ from particle tracking we find this time to be $>30 \mathrm{~s}$. Fig. 2 shows that fluctuations in jamming and unjamming (or the near-periodic variation seen at $\phi=0.505$ ) occur at a rate similar or faster than this. In other words, at slower flows, force chains are broken up by fluctuations faster than they are bodily rotated by the turn in the flow. Hence vortices are never established. What causes the fluctuationdriven breakup of jams remains unclear. It may relate to the role of fluid permeation ${ }^{15}$ (see above) or local particle diffusion: again, current work is underway to elucidate the role of the fluid phase and understand better the jam-unjam behaviour.

\section{Conclusions}

Here we have demonstrated that at the lowest applied pressure $\left(v_{1}\right)$ and $\phi \leqslant 0.50$, the mean flow rate $\langle V\rangle$ of the dispersions as they are drawn into the capillary is approximately proportional to the pressure drop ( $\equiv$ pump speed) and inversely proportional to viscosity $\eta$; i.e. they follow Hagen-Poiseuille flow for a simple liquid. When $\phi \geqslant 0.505$ granular-like jamming behaviour is observed with $\langle V\rangle$ becoming independent of pressure drop. At the highest applied pressure $\left(v_{2}\right)$ the system is able to 'permanently' unjam due to the formation of rotating vortices in the spatial flow pattern, thus reverting from granular-like behaviour back to a simple hard-sphere liquid like system. In the experiment here the important role played by the geometry in imposing a rotation on the flow pattern, thus rotating force chains and taking advantage of the 'fragility' of the jammed system, suggests a simple technical solution to jamming problems in processes involving channels: insert similar 'turns' in straight channels to enable the jamming fluid to self-lubricate and revert to simple viscous flow.

\section{Acknowledgements}

We thank Dr Nadeem Javid for sizing the PMMA particles, Dr Andrew Schofield for providing the particles and the EPSRC (grant EP/E043976) for financial support.

\section{References}

1 M. E. Cates, J. P. Wittmer, J. P. Bouchaud and P. Claudin, Phys. Rev. Lett., 1998, 81, 1841-1844.

2 C. Mankoc, A. Garcimartin, I. Zuriguel, D. Maza and L. A. Pugnaloni, Phys. Rev. E: Stat., Nonlinear, Soft Matter Phys, 2009, 80, 011309. 
3 M. T. Roberts, A. Mohraz, K. T. Christensen and J. A. Lewis, Langmuir, 2007, 23, 8726-8731.

4 R. Karlsson, M. Karlsson, A. Karlsson, A. S. Cans, J. Bergenholtz, B. Akerman, A. G. Ewing, M. Voinova and O. Orwar, Langmuir, 2002, 18, 4186-4190.

5 M. D. Haw, Phys. Rev. Lett., 2004, 92, 185506.

6 S. P. Meeker, W. C. K. Poon and P. N. Pusey, Phys. Rev. E: Stat. Phys., Plasmas, Fluids, Relat. Interdiscip. Top., 1997, 55, 57185722.

7 W. J. Frith, P. dHaene, R. Buscall and J. Mewis, J. Rheol., 1996, 40, 531-548.

8 D. Lootens, H. Van Damme and P. Hebraud, Phys. Rev. Lett., 2003, 90, 178301
9 F. Alonso-Marroquin, I. Vardoulakis, H. J. Herrmann, D. Weatherley and P. Mora, Phys. Rev. E: Stat., Nonlinear, Soft Matter Phys., 2006, 74, 031306.

10 P. N. Pusey and W. Vanmegen, Nature, 1986, 320, 340-342.

11 W. C. K. Poon, S. P. Meeker, P. N. Pusey and P. N. Segre, J. NonNewtonian Fluid Mech., 1996, 67, 179-189.

12 P. N. Segre, S. P. Meeker, P. N. Pusey and W. C. K. Poon, Phys. Rev. Lett., 1995, 75, 958-961.

13 J. Crocker and D. Grier, J. Colloid Interface Sci., 1996, 179, 298-310.

14 A. I. Campbell, R. A. W. Dryfe and M. D. Haw, Anal. Sci., 2009, 25, $307-310$.

15 L. Isa, R. Besseling, A. N. Morozov and W. C. K. Poon, Phys. Rev. Lett., 2009, 102, 058302. 